\title{
Inclusion Relationship of Uncertain Sets
}

Correspondence: yaokai@ucas.ac.cn School of Economics and

Management, University of Chinese Academy of Sciences, Beijing 100190 China

\section{Springer}

\begin{abstract}
Uncertain set is a set-valued function on an uncertainty space, and membership function is used to describe an uncertain set. This paper discusses the inclusion relationship between two uncertain sets and illustrates the relationship via some examples. A formula is derived to calculate the uncertain measure that an uncertain set is included in another one.
\end{abstract}

Keywords: Uncertain set; Membership function; Uncertainty theory; Inclusion relationship

\section{Introduction}

In order to indicate human's belief degree on the chance that an event happens, an uncertain measure was defined by Liu $[1,2]$ as a set function satisfying normality, duality, subadditivity and product axioms. Uncertain set, initialized by Liu [3], is a set-valued function on an uncertainty space to model the unsharp concepts such as "old people" and "heavy vehicles". In order to describe an uncertain set, a concept of membership function was proposed by Liu [4].

Uncertain sets are the theoretical foundations of uncertain inference control and uncertain logic. In 2010, Liu [3] proposed some inference rules for uncertain systems, which were extended to the rules with multiple antecedents by Gao et al. [5]. Following that, Gao [6] balanced an inverted pendulum by using the uncertain controller. In addition, Peng and Chen [7] showed that the uncertain inference system is a universal approximator. In 2011, Liu [8] applied uncertain set to uncertain logic for dealing with human language by using the truth value formula of uncertain propositions.

The concept of independence for uncertain sets was defined by Liu [9]. Then, the set operational law and arithmetic operational law of independent uncertain sets were given by Liu [4]. The concept of expected value for uncertain set was defined by Liu [3], and formulas to calculate the expected value using the membership function and inverse membership function were given by Liu [10] and Liu [4], respectively. Besides, the variance of uncertain set was proposed by Liu [8] and was investigated by Yang and Gao [11]. In addition, the entropy of uncertain set was presented by Liu [4] and was further studied by Yao and Ke [12], Yao [13], Wang and Ha [14], and Peng and Li [15]. For more details about uncertain sets, please refer to Liu [16] and Yang and Gao [17].

In this paper, we will study the inclusion relationship of uncertain sets. The rest of this paper is organized as follows. The next section is intended to introduce some basic concepts about uncertain sets. Then the inclusion relationship is introduced in

(c) 2015 Yao. Open Access This article is distributed under the terms of the Creative Commons Attribution 4.0 International License (http://creativecommons.org/licenses/by/4.0/), which permits unrestricted use, distribution, and reproduction in any medium, provided you give appropriate credit to the original author(s) and the source, provide a link to the Creative Commons license, and indicate if changes were made. 
Section "Inclusion Relationship", and a formula to calculate the uncertain measure that an uncertain set is included in another one is derived in Section "Main Results". Finally, some conclusions are made in Section "Conclusions".

\section{Preliminary}

This section introduces some results about uncertain sets, including the uncertainty space, membership function, independence, and the set operational law.

Definition 1. (Liu [1]) Let $\Gamma$ be a nonempty set, and $\mathcal{L}$ be a $\sigma$-algebra on $\Gamma$. A set function $\mathcal{M}$ is called an uncertain measure if it satisfies the following axioms,

Axiom 1: (Normality) $\mathcal{M}\{\Gamma\}=1$;

Axiom 2: (Duality) $\mathcal{M}\{\Lambda\}+\mathcal{M}\left\{\Lambda^{c}\right\}=1$ for any $\Lambda \in \mathcal{L}$;

Axiom 3: (Subadditivity) For every sequence of $\left\{\Lambda_{i}\right\} \in \mathcal{L}$, we have

$$
\mathcal{M}\left\{\bigcup_{i=1}^{\infty} \Lambda_{i}\right\} \leq \sum_{i=1}^{\infty} \mathcal{M}\left\{\Lambda_{i}\right\}
$$

In this case, the triple $(\Gamma, \mathcal{L}, \mathcal{M})$ is called an uncertainty space.

Besides, a product axiom on the product uncertainty space was given by Liu [2] for calculating uncertain sets in 2009.

Axiom 4: (Product Axiom) Let $\left(\Gamma_{k}, \mathcal{L}_{k}, \mathcal{M}_{k}\right)$ be uncertainty spaces for $k=1,2, \ldots$

Then the product uncertain measure $\mathcal{M}$ is an uncertain measure satisfying

$$
\mathcal{M}\left\{\prod_{i=1}^{\infty} \Lambda_{k}\right\}=\bigwedge_{k=1}^{\infty} \mathcal{M}_{k}\left\{\Lambda_{k}\right\}
$$

where $\Lambda_{k}$ are arbitrarily chosen events from $\mathcal{L}_{k}$ for $k=1,2, \cdots$, respectively.

Definition 2. (Liи [3]) An uncertainty set is a measurable function $\xi$ from an uncertainty space $(\Gamma, \mathcal{L}, \mathcal{M})$ to a collection of sets of real numbers, i.e., for any Borel set $B$ of real numbers, the following two sets

$$
\begin{aligned}
& \{\xi \subset B\}=\{\gamma \in \Gamma \mid \xi(\gamma) \subset B\} \\
& \{B \subset \xi\}=\{\gamma \in \Gamma \mid B \subset \xi(\gamma)\}
\end{aligned}
$$

are events.

An uncertain set $\xi$ is called nonempty if $\mathcal{N}\{\xi=\emptyset\}=0$.

Definition 3. (Liu [4]) An uncertain set $\xi$ is said to have a membership function $\mu$ if the equations

$$
\begin{aligned}
& \mathcal{M}\{B \subset \xi\}=\inf _{x \in B} \mu(x) \\
& \mathcal{M}\{\xi \subset B\}=1-\sup _{x \in B^{c}} \mu(x)
\end{aligned}
$$

hold for any Borel set $B$ of real numbers.

Definition 4. (Liu [9]) The uncertain sets $\xi_{1}, \xi_{2}, \ldots, \xi_{n}$ are said to be independent if for any Borel sets of real numbers, we have

$$
\mathcal{M}\left\{\bigcap_{i=1}^{n}\left(\xi_{i}^{*} \subset B_{i}\right)\right\}=\bigwedge_{i=1}^{n} \mathcal{M}\left\{\xi_{i}^{*} \subset B_{i}\right\}, \quad \mathcal{M}\left\{\bigcup_{i=1}^{n}\left(\xi_{i}^{*} \subset B_{i}\right)\right\}=\bigvee_{i=1}^{n} \mathcal{M}\left\{\xi_{i}^{*} \subset B_{i}\right\},
$$

where $\xi_{i}^{*}$ are arbitrarily chosen from $\left\{\xi_{i}, \xi_{i}^{c}\right\}, i=1,2, \ldots, n$, respectively. 
Theorem 1. (Liu [4]) Let $\xi$ and $\eta$ be independent uncertain sets with membership functions $\mu$ and $\nu$, respectively. Then the union $\xi \cup \eta$ has a membership function $\mu(x) \vee \nu(x)$, the intersection $\xi \cap \eta$ has a membership function $\mu(x) \wedge v(x)$, and the complement $\xi^{c}$ has a membership function $1-\mu(x)$.

\section{Inclusion Relationship}

In this section, we illustrate the inclusion relationship between uncertain sets via some examples.

Example 1. Consider an uncertainty space $(\Gamma, \mathcal{L}, \mathcal{M})$ with $\Gamma=\left\{\gamma_{1}, \gamma_{2}, \gamma_{3}\right\}, \mathcal{M}\left\{\gamma_{1}\right\}=$ $0.3, \mathcal{M}\left\{\gamma_{2}\right\}=0.4$ and $\mathcal{M}\left\{\gamma_{3}\right\}=0.5$. Define two uncertain sets

$$
\xi(\gamma)=\left\{\begin{array}{ll}
{[0,1],} & \text { if } \gamma=\gamma_{1} \\
{[-2,2],} & \text { if } \gamma=\gamma_{2} \\
{[0,3],} & \text { if } \gamma=\gamma_{3},
\end{array} \quad \eta(\gamma)=\left\{\begin{array}{l}
{[-1,1], \text { if } \gamma=\gamma_{1}} \\
{[0,2], \text { if } \gamma=\gamma_{2}} \\
{[-3,0], \text { if } \gamma=\gamma_{3} .}
\end{array}\right.\right.
$$

Then

$$
\mathcal{M}\{\xi \subset \eta\}=\mathcal{M}\{\gamma \in \Gamma \mid \xi(\gamma) \subset \eta(\gamma)\}=\mathcal{M}\left\{\gamma_{1}\right\}=0.3
$$

and

$$
\mathcal{M}\{\eta \subset \xi\}=\mathcal{M}\{\gamma \in \Gamma \mid \eta(\gamma) \subset \xi(\gamma)\}=\mathcal{M}\left\{\gamma_{2}\right\}=0.4 .
$$

Example 2. Let $\emptyset$ be the empty set, and let $\xi$ be a nonempty uncertain set. Then

$$
\mathcal{M}\{\emptyset \subset \xi\}=1
$$

and

$$
\mathcal{M}\{\xi \subset \varnothing\}=0 .
$$

Example 3. Let $\xi$ be an uncertain set, and let $\Re$ be the set of real numbers. Then

$$
\mathcal{M}\{\xi \subset \mathfrak{R}\}=1 .
$$

Example 4. Consider an uncertainty space $(\Gamma, \mathcal{L}, \mathcal{N})$ with $\Gamma=\left\{\gamma_{1}, \gamma_{2}\right\}$ and $\mathcal{M}\left\{\gamma_{1}\right\}=c$ and $\mathcal{M}\left\{\gamma_{2}\right\}=1-c$. Define an uncertain set

$$
\xi(\gamma)=\left\{\begin{array}{l}
\Re, \text { if } \gamma=\gamma_{1} \\
\emptyset, \text { if } \gamma=\gamma_{2} .
\end{array}\right.
$$

Then we have

$$
\mathcal{M}\{\xi \subset \emptyset\}=1-c
$$

and

$$
\mathcal{M}\{\Re \subset \xi\}=c .
$$

\section{Main Results}

In this section, we derive a formula to calculate the uncertain measure that an uncertain set is included in another one. 
Theorem 2. Let $\xi$ and $\eta$ be two independent uncertain sets with membership functions $\mu$ and $v$, respectively. Then we have

$$
\mathcal{M}\{\xi \subset \eta\}=\inf _{x \in \Re^{*}}(1-\mu(x)) \vee v(x) .
$$

Proof. Since

$$
\{\gamma \in \Gamma \mid \xi(\gamma) \subset \eta(\gamma)\}=\left\{\gamma \in \Gamma \mid \xi(\gamma) \cap \eta^{c}(\gamma)=\emptyset\right\},
$$

we have

$$
\mathcal{M}\{\xi \subset \eta\}=\mathcal{M}\left\{\xi \cap \eta^{c}=\emptyset\right\}=\mathcal{M}\left\{\xi \xi \cap \eta^{c} \subset \emptyset\right\} .
$$

Since $\xi \cap \eta^{c}$ has a membership function $\mu(x) \wedge(1-v(x))$, it follows from the definition of membership function that

$$
\mathcal{N}\left\{\xi \cap \eta^{c} \subset \emptyset\right\}=1-\sup _{x \in \emptyset^{c}} \mu(x) \wedge(1-v(x))=\inf _{x \in \Re}(1-\mu(x)) \vee v(x) .
$$

The theorem is proved.

Remark 1. To my best knowledge, there is not such a formula in the fuzzy set theory to calculate the possibility measure that a fuzzy set is included in another one.

Example 5. Let $\xi$ be an uncertain set with a triangular membership function $(-4,0,4)$, and let $\eta$ be an uncertain set with a trapezoidal membership function $(-2,-1,1,2)$. If $\xi$ and $\eta$ are independent, then

$$
\mathcal{N}\{\xi \subset \eta\}=0.4
$$

and

$$
\mathcal{M}\{\eta \eta \subset \xi\}=0.6 .
$$

Example 6. Let $\xi$ be an uncertain set with a membership function $\mu(x)$, and $B$ be a Borel set. Note that $B$ can be regarded as an uncertain set with a membership function $1_{B}(x)$, and it is independent of the uncertain set $\xi$. Then according to Theorem 2 , we have

$$
\mathcal{M}\{B \subset \xi\}=\inf _{x \in \Re}\left(1-1_{B}(x)\right) \vee \mu(x)=\inf _{x \in B} \mu(x)
$$

and

$$
\mathcal{N}\{\xi \subset B\}=\inf _{x \in \Re}(1-\mu(x)) \vee 1_{B}(x)=\inf _{x \in B^{c}}(1-\mu(x))=1-\sup _{x \in B^{c}} \mu(x),
$$

which coincide with Definition 3 of the membership function.

Example 7. Let $B_{1}=[1,3]$ and $B_{2}=[2,4]$ be two sets of real numbers. Note that they could be regarded as two independent uncertain sets with membership functions $1_{B_{1}}(x)$ and $1_{B_{2}}(x)$, respectively. Then we have

$$
\mathcal{M}\{[1,3] \subset[2,4]\}=\mathcal{M}\left\{B_{1} \subset B_{2}\right\}=\inf _{x \in \Re}\left(1-1_{B_{1}}(x)\right) \vee 1_{B_{2}}(x)=0
$$

and

$$
\mathcal{N}\{[2,4] \subset[1,3]\}=\mathcal{N}\left\{B_{2} \subset B_{1}\right\}=\inf _{x \in \Re} 1_{B_{1}}(x) \vee\left(1-1_{B_{2}}(x)\right)=0,
$$

which coincide with the inclusion relationship of classic sets. 


\section{Conclusions}

This paper mainly studied the inclusion relationship function of uncertain sets. It gave a formula to calculate the uncertain measure that an uncertain set is included in another one when they are independent.

\section{Competing interests}

The authors declare that they have no competing interests.

\section{Acknowledgements}

This work was supported by National Natural Science Foundation of China (Grant No. 61403360).

Received: 26 August 2015 Accepted: 30 August 2015

Published online: 21 October 2015

\section{References}

1. Liu, B: Uncertainty Theory. 2nd edn, Berlin (2007), Springer-Verlag

2. Liu, B: Some research problems in uncertainty theory. J. Uncertain Syst. 3(1), 3-10 (2009)

3. Liu, B: Uncertain set theory and uncertain inference rule with application to uncertain control. J. Uncertain Syst. 4(2), 83-98 (2010)

4. Liu, B: Membership functions and operational law of uncertain sets. Fuzzy Optim. Decis. Making. 11(4), 387-410 (2012)

5. Gao, X, Gao, Y, Ralescu, DA: On Liu's inference rule for uncertain systems. Int. J. Uncertainty Fuzziness Knowledge-Based Syst. 18(1), 1-11 (2010)

6. Gao, Y: Uncertain inference control for balancing inverted pendulum. Fuzzy Optim. Decis. Making. 11(4), 481-492 (2012)

7. Peng, Z, Chen, X: Uncertain systems are universal approximators. J. Uncertainty Anal. Appl. 2(13) (2014)

8. Liu, B: Uncertain logic for modeling human language. J. Uncertain Syst. 5(1), 3-20 (2011)

9. Liu, B: A new definition of independence of uncertain sets. Fuzzy Optim. Decis. Making. 12(4), 451-461 (2013)

10. Liu, B: Uncertainty Theory: A branch of mathematics for modeling human uncertainty. Springer-Verlag, Berlin (2010)

11. Yang, XF, Gao, J: Some results of moments of uncertain set. J. Intel. Fuzzy Syst. 28(6), 2433-2442 (2015)

12. Yao, K, Ke, H: Entropy operator for membership function of uncertain set. Appl. Math. Comput. 242, 898-906 (2014)

13. Yao, K: Sine entropy of uncertain set and its applications. Appl. Soft Comput. 22, 432-442 (2014)

14. Wang, XS, Ha, MH: Quadratic entropy of uncertain sets. Fuzzy Optim. Decis. Making. 12(1), 99-109 (2013)

15. Peng, J, Li, SG: Radical entropies of uncertain sets. Inf. Int. Interdiscip. J. 16(2A), 895-902 (2013)

16. Liu, B: Uncertainty Theory. 4th edn. Springer-Verlag, Berlin (2015)

17. Yang, XF, Gao, J: A review on uncertain set. J. Uncertain Syst. 8(4), 285-300 (2014)

\section{Submit your manuscript to a SpringerOpen ${ }^{0}$ journal and benefit from:}

- Convenient online submission

- Rigorous peer review

- Immediate publication on acceptance

- Open access: articles freely available online

- High visibility within the field

- Retaining the copyright to your article

Submit your next manuscript at $\mathbf{s p r i n g e r o p e n . c o m ~}$ 